\title{
Correlating the Structure and Optical Absorption Properties of $\mathrm{Au}_{76}(\mathrm{SR})_{44}$ Cluster
}

\author{
Zhongyun $\mathrm{Ma}^{\mathrm{a}}$, Pu Wang ${ }^{\mathrm{a}}$, Guang Zhou ${ }^{\mathrm{b}}$, Jian Tang ${ }^{\mathrm{b}}$, Hengfeng $\mathrm{Li}^{\mathrm{c}}$ and Yong Pei*a \\ ${ }^{a}$ Department of Chemistry, Key Laboratory of Environmentally Friendly Chemistry and \\ Applications of Ministry of Education, Xiangtan University, Hunan Province, Xiangtan 411105, P. \\ R. China \\ ${ }^{b}$ Hunan Key Laboratory for Computation and Simulation in Science and Engineering, Institute for \\ Computational and Applied Mathematics, Xiangtan University, Hunan Province, Xiangtan 411105, \\ P. R. China \\ ${ }^{c}$ School of Materials Science and Engineering, Central South University, Hunan Province, \\ Changsha 410083, P. R. China
}




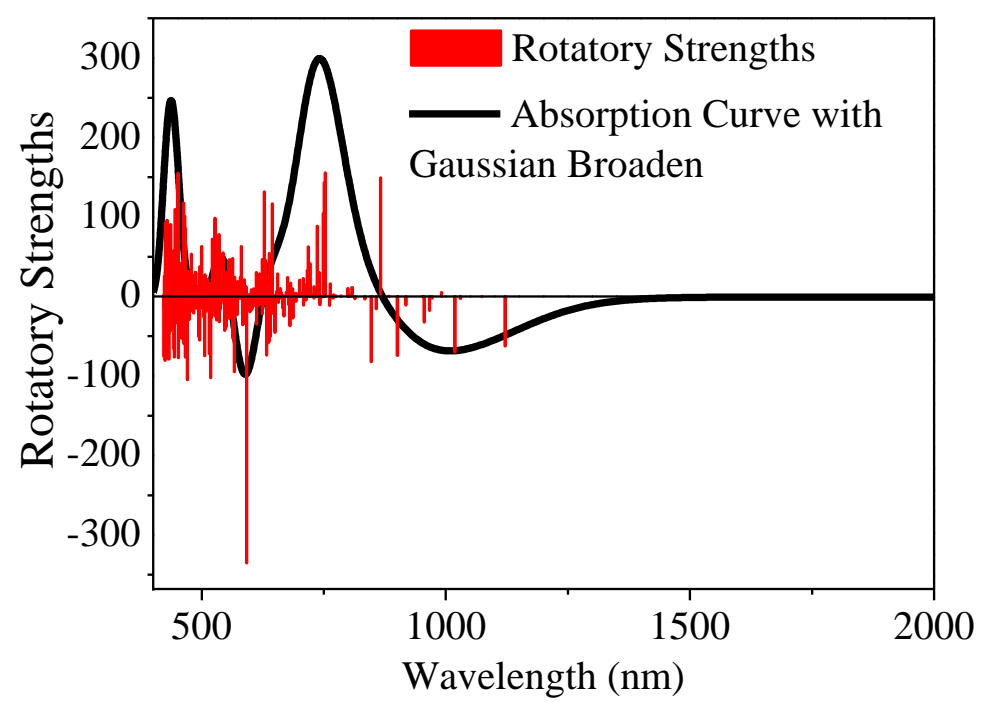

Figure S1. Simulated CD spectra of $\mathrm{Au}_{76}(\mathrm{SH})_{44}$ cluster. The rotatory strength is in unit of $10^{-40} \mathrm{esu}^{2} \mathrm{~cm}^{2}$.

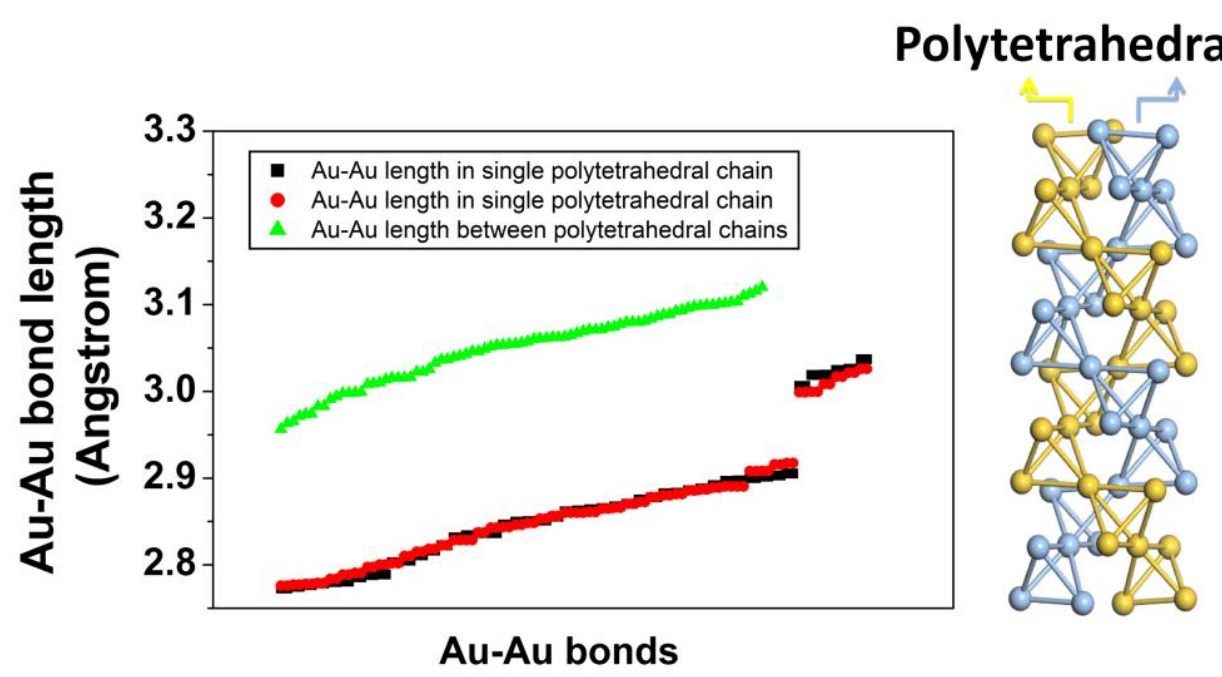

Figure S2. $\mathrm{Au}-\mathrm{Au}$ bond lengths in the $\mathrm{Au}_{50}$-core of $\mathrm{Au}_{76}(\mathrm{SH})_{44}$. 


\begin{tabular}{|c|c|c|c|}
\hline \multicolumn{4}{|c|}{ Coordinates of $\mathrm{Au}_{76}(\mathrm{SH})_{44}$} \\
\hline $\mathrm{Au}$ & -6.479254402 & -0.071625628 & -4.957875120 \\
\hline $\mathrm{Au}$ & -9.092817685 & 5.161889726 & -0.040901455 \\
\hline$u$ & -9.039008063 & -5.195512202 & 0.006149712 \\
\hline lu & -8.966033231 & 1.851007768 & 3.131725710 \\
\hline $\mathrm{Au}$ & 8.948640025 & -1.860794681 & 3.150281711 \\
\hline lu & 10.397253113 & 3.619799378 & 3.119309871 \\
\hline lu & 10.383652880 & -3.618270432 & 92244 \\
\hline u & 8.960537730 & 1.866129783 & -3.126052480 \\
\hline $\mathrm{Au}$ & -8.957025874 & -1.855588874 & -3.134 \\
\hline lu & 5959475 & 3.63809 & 4193 \\
\hline$u$ & -10.364414783 & 428 & 2030 \\
\hline$u$ & -6.479634438 & 745 & 866 \\
\hline$A u$ & 6.468432020 & -0.07 & 6660 \\
\hline$u$ & & 148 & 8240 \\
\hline$u$ & & & 052 \\
\hline $\mathrm{Au}$ & 6.480456184 & 943 & 841 \\
\hline$S$ & 7771379 & 95 & -5. \\
\hline ; & -7.5 & & 089 \\
\hline 5 & 3436965 & -5.0 & -1.77 \\
\hline 5 & 3767495 & 749 & 0684 \\
\hline$S$ & 383 & -1.7 & 058 \\
\hline & & & 2035 \\
\hline & 10.204846730 & -1.8 & 4907 \\
\hline & 3735 & 45 & 092 \\
\hline & & & \\
\hline & 817414 & 602 & 207 \\
\hline$\sigma$ & 560702 & -1.8 & 514 \\
\hline$S$ & & & \\
\hline > & & & 2402 \\
\hline$c$ & 2048179 & & 3539 \\
\hline S & 9717456 & -5.0 & 1158 \\
\hline$S$ & & & 6737 \\
\hline$c$ & -7.680034729 & -2.1 & -5. \\
\hline S & -10.756702268 & 5.429496993 & 5921 \\
\hline$S$ & -10.658989820 & -5.480734241 & 1.690665880 \\
\hline & -7.680159284 & & 5.097937747 \\
\hline$S$ & 7.650733297 & -2.111368337 & 5.110153246 \\
\hline$S$ & & & 1.684361057 \\
\hline$S$ & 10.682152553 & -5.461034347 & -1.687158708 \\
\hline$S$ & & & -5.095346793 \\
\hline $\mathrm{Au}$ & -6.513166783 & -0.103850492 & -1.508510040 \\
\hline $\mathrm{Au}$ & -6.497303610 & 0.095899438 & 1.495167522 \\
\hline $\mathrm{Au}$ & -6.588988234 & 2.742176288 & -1.664379693 \\
\hline
\end{tabular}




\begin{tabular}{|c|c|c|c|}
\hline $\mathrm{Au}$ & -6.554833324 & -2.740877543 & 1.721225139 \\
\hline $\mathrm{Au}$ & -8.614293789 & -1.500564011 & 0.281801200 \\
\hline & 8.619832922 & 1.464285060 & -0.274584161 \\
\hline & 8.466617815 & 1.132541918 & -3.053824564 \\
\hline & 8.440725853 & -1.116861831 & 3.065423491 \\
\hline & -6.446968233 & -2.915035098 & -1.346730821 \\
\hline & 6.448834419 & 2.914010137 & 1.343359729 \\
\hline & 2.171852632 & -0.020150978 & 732571 \\
\hline & -2.160641880 & 0.018649431 & 1.511540710 \\
\hline & -2.287500570 & 2.855764622 & -1.514573541 \\
\hline & -2.294444316 & -2.856248729 & 920837 \\
\hline & 5113683 & -1.51385 & 0.04 \\
\hline & -4.335500645 & 1.483859234 & 190819 \\
\hline & -4.507981175 & -1.589989010 & 157793 \\
\hline & 1864217 & 1.39160 & 958484 \\
\hline & 3164859 & $1.5830^{\circ}$ & 488417 \\
\hline & 5922561 & 4.871826828 & 378303 \\
\hline & 1596277 & -1.414786918 & 2.940966586 \\
\hline & 2953975 & -4.86652 & 0.08 \\
\hline & & & \\
\hline & -5.723245433 & -5.002827826 & 2.072794465 \\
\hline & -5.703235162 & 4.9778 & $-2.0<$ \\
\hline & & & \\
\hline & -3.427958675 & -2.076596872 & 512856 \\
\hline & -3.205472487 & -5.00191 & 477109 \\
\hline & -3.429924040 & 2.0 & \\
\hline & -2.054504345 & -2.87770 & -1.458209907 \\
\hline & -2.029690145 & 2.902469965 & 1.430577870 \\
\hline & 2.163290715 & 0.014289314 & 019293 \\
\hline & 2.163259255 & -0.0 & 381 \\
\hline & -2.185403458 & -0.068896115 & -4.864377391 \\
\hline & 2.041386414 & 2.881341388 & -1.442907931 \\
\hline & 2.034427088 & -2.90201 & 1.42837 \\
\hline & 0.000203648 & -1.48310 & 0.003674340 \\
\hline $\mathrm{Au}$ & 0.001399602 & 1.490614329 & 0.003900431 \\
\hline & -0.126307098 & -1.475543479 & -2.884152410 \\
\hline & 0.127706982 & 1.488099037 & -2.890866164 \\
\hline & -0.124154248 & 1.492840735 & 2.883776321 \\
\hline $\mathrm{Au}$ & 0.001553489 & 4.871706329 & -0.003132258 \\
\hline & 0.126815750 & -1.487832275 & 2.881169320 \\
\hline & -0.004199234 & -4.868805836 & -0.008070434 \\
\hline $\mathrm{Au}$ & 2.186039167 & -0.074170655 & 4.854839522 \\
\hline$S$ & -0.946971583 & 1.941240891 & -5.050492651 \\
\hline$S$ & -1.188038184 & -4.995361300 & 2.03455475 \\
\hline
\end{tabular}




\begin{tabular}{|c|c|c|c|}
\hline$S$ & -1.189278414 & 4.996717822 & -2.043265184 \\
\hline$S$ & 1.190312194 & 4.998323933 & 2.038437343 \\
\hline S & 0.933627367 & -1.926359753 & -5.053324697 \\
\hline$S$ & 1.182847128 & -4.999243906 & -2.048679575 \\
\hline S & -0.928278098 & -1.941675744 & 5.048620743 \\
\hline S & 0.937140363 & 1.930122347 & 5.051119123 \\
\hline $\mathrm{Au}$ & 2.289402302 & -2.866138367 & -1.523824444 \\
\hline $\mathrm{Au}$ & 2.305637830 & 2.865495678 & 1.510845461 \\
\hline $\mathrm{Au}$ & 6.505082683 & 0.102666930 & -1.487461662 \\
\hline $\mathrm{Au}$ & 6.499145888 & -0.102971261 & 1.514948564 \\
\hline $\mathrm{Au}$ & 2.181991616 & 0.077956111 & -4.866971282 \\
\hline $\mathrm{Au}$ & 6.448306425 & 2.917644825 & -1.358939849 \\
\hline $\mathrm{Au}$ & 6.440106222 & -2.917381724 & 1.306537014 \\
\hline $\mathrm{Au}$ & 4.322985994 & -1.485279623 & -0.056525474 \\
\hline $\mathrm{Au}$ & 4.338272362 & 1.495961367 & 0.049542935 \\
\hline $\mathrm{Au}$ & 4.189703500 & -1.395503093 & -2.938036306 \\
\hline $\mathrm{Au}$ & 4.487032586 & 1.586572128 & -2.832060480 \\
\hline $\mathrm{Au}$ & 4.191988687 & 1.403499598 & 2.934993684 \\
\hline $\mathrm{Au}$ & 4.446893846 & 4.867779353 & 0.082386424 \\
\hline $\mathrm{Au}$ & 4.471784006 & -1.603544578 & 2.812626033 \\
\hline $\mathrm{Au}$ & 4.431746748 & -4.880409898 & -0.072618029 \\
\hline S & 3.434927634 & 2.080116563 & -4.988334187 \\
\hline$S$ & 3.187581085 & -5.016574185 & 1.930341174 \\
\hline$S$ & 3.201298632 & 5.000294008 & -1.921708415 \\
\hline$S$ & 5.733524607 & 4.991684922 & 2.065650690 \\
\hline S & 5.715385040 & -4.986894342 & -2.056404808 \\
\hline$S$ & 3.432028116 & -2.080362973 & 4.978647839 \\
\hline $\mathrm{Au}$ & 6.570176483 & -2.731974853 & -1.707457313 \\
\hline $\mathrm{Au}$ & 6.574017844 & 2.736883917 & 1.695456856 \\
\hline $\mathrm{Au}$ & 8.624988604 & -1.490741181 & -0.271110734 \\
\hline $\mathrm{Au}$ & 8.611632012 & 1.466124101 & 0.283489671 \\
\hline $\mathrm{Au}$ & 8.455925178 & -1.106197590 & -3.049663535 \\
\hline $\mathrm{Au}$ & 8.450566168 & 1.120924538 & 3.068658862 \\
\hline $\mathrm{H}$ & -11.134272224 & 1.105879368 & -4.213003534 \\
\hline $\mathrm{H}$ & -10.118584682 & 6.386247052 & -2.414052548 \\
\hline $\mathrm{H}$ & -8.434425818 & 4.632502242 & 2.667155620 \\
\hline $\mathrm{H}$ & -10.523412938 & 3.121252482 & 1.105363811 \\
\hline $\mathrm{H}$ & -8.633536261 & 1.672637460 & 5.966011672 \\
\hline $\mathrm{H}$ & -6.194903464 & -2.740399300 & 4.652711793 \\
\hline $\mathrm{H}$ & -11.176671417 & -1.083229233 & 4.060637809 \\
\hline $\mathrm{H}$ & -9.970433274 & -6.387483466 & 2.437962233 \\
\hline $\mathrm{H}$ & -8.409155589 & -4.628034305 & -2.705913204 \\
\hline $\mathrm{H}$ & -10.498095603 & -3.167560055 & -1.127104045 \\
\hline $\mathrm{H}$ & -8.637360060 & -1.647181864 & -5.966359955 \\
\hline
\end{tabular}




\begin{tabular}{lrrr}
$\mathrm{H}$ & -6.190869177 & 2.754667351 & -4.609196037 \\
$\mathrm{H}$ & -6.779152373 & 5.634405998 & -1.516707737 \\
$\mathrm{H}$ & -2.190581266 & 5.816494517 & 1.478918737 \\
$\mathrm{H}$ & -2.461817573 & 2.892397464 & 4.495928462 \\
$\mathrm{H}$ & -1.891402096 & -2.778633536 & 4.568766618 \\
$\mathrm{H}$ & -6.812776856 & -5.618009534 & 1.514085001 \\
$\mathrm{H}$ & -2.198538524 & -5.795394050 & -1.447912611 \\
$\mathrm{H}$ & -2.463592779 & -2.897542137 & -4.491787076 \\
$\mathrm{H}$ & -1.912324587 & 2.769632967 & -4.560692994 \\
$\mathrm{H}$ & -2.208864705 & 5.783047779 & -1.588362606 \\
$\mathrm{H}$ & 2.208631889 & 5.788995648 & 1.588772654 \\
$\mathrm{H}$ & 2.199895683 & 5.799884378 & -1.448811264 \\
$\mathrm{H}$ & 6.817475178 & 5.620223530 & 1.513389817 \\
$\mathrm{H}$ & 8.428441220 & 4.637615974 & -2.682311343 \\
$\mathrm{H}$ & 10.065354753 & 6.373671834 & 2.427075923 \\
$\mathrm{H}$ & 11.171612218 & 1.063919396 & 4.097833372 \\
$\mathrm{H}$ & 6.187660087 & 2.756262760 & 4.623870366 \\
$\mathrm{H}$ & 1.902247482 & 2.766249361 & 4.574071685 \\
$\mathrm{H}$ & 2.459711593 & -2.906127929 & 4.498363466 \\
$\mathrm{H}$ & -2.209503802 & -5.782721706 & 1.585444191 \\
$\mathrm{H}$ & 2.200941223 & -5.788677455 & -1.595969235 \\
$\mathrm{H}$ & 2.186545810 & -5.816806112 & 1.458207435 \\
$\mathrm{H}$ & 6.802699713 & -5.614828855 & -1.509713736 \\
$\mathrm{H}$ & 8.400379986 & -4.611511121 & 2.696805680 \\
$\mathrm{H}$ & 9.997604466 & -6.369363309 & -2.436636089 \\
$\mathrm{H}$ & 11.191366432 & -1.059522574 & -4.045488362 \\
$\mathrm{H}$ & 1.898213517 & -2.760886950 & -4.572378263 \\
$\mathrm{H}$ & 2.469402431 & 2.906549606 & -4.495772533 \\
$\mathrm{H}$ & 6.196968012 & -2.728084041 & -4.637228645 \\
& 8.636591833 & 1.684976871 & -5.958171720 \\
$\mathrm{H}$ & 10.507153906 & 3.138022956 & -1.091356062 \\
\hline & 8.603670253 & -1.675933178 & 5.982799482
\end{tabular}

